\title{
Internet-delivered exposure-based
} cognitive-behavioural therapy and behavioural stress management for severe health anxiety: randomised controlled trial

Erik Hedman, Erland Axelsson, Anders Görling, Carina Ritzman, Markus Ronnheden, Samir El Alaoui, Erik Andersson, Mats Lekander and Brjánn Ljótsson

\section{Background}

Exposure-based cognitive-behavioural therapy (CBT) delivered via the internet has been shown to be effective for severe health anxiety (hypochondriasis) but has not been compared with an active, effective and credible psychological treatment, such as behavioural stress management (BSM)

\section{Aims \\ To investigate two internet-delivered treatments - exposure-based CBT v. BSM - for severe health anxiety in a randomised controlled trial (trial registration: NCT01673035).}

\section{Method}

Participants $(n=158)$ with a principal diagnosis of severe health anxiety were allocated to 12 weeks of exposure-based
CBT $(n=79)$ or BSM $(n=79)$ delivered via the internet. The Health Anxiety Inventory (HAl) was the primary outcome.

\section{Results}

Internet-delivered exposure-based CBT led to a significantly greater improvement on the HAl compared with BSM. However, both treatment groups made large improvements on the HAl (pre-to-post-treatment Cohen's d: exposure-based CBT, 1.78; BSM, 1.22)

\section{Conclusions}

Exposure-based CBT delivered via the internet is an efficacious treatment for severe health anxiety.

\section{Declarations of interest}

None.
Severe health anxiety, or hypochondriasis according to DSM-IV, is common in medical clinics, follows a chronic course, and leads to functional impairment and suffering. ${ }^{2-4}$ Cognitive-behavioural therapy (CBT) has been shown to be effective in randomised trials in primary and secondary medical settings. ${ }^{5-7}$ It generally comprises a broad spectrum of treatments including exposure-based therapy, cognitive therapy and applied relaxation. ${ }^{8}$ Exposure-based therapy is thought to lead to extinction of health anxiety symptoms through repeated contact with stimuli that trigger the symptoms (e.g. to fully experience bodily sensations while refraining from seeking medical reassurance). ${ }^{9}$ By contrast, applied relaxation is a method used to directly control symptoms through counteracting early signs of anxiety with relaxation techniques. ${ }^{10}$ Although two studies ${ }^{5,11}$ have shown that cognitive therapy can be superior to active psychological treatments - such as behavioural stress management (BSM), which comprises applied relaxation and stress management, and psychodynamic therapy - no study has demonstrated superiority of exposure-based CBT to an active psychological treatment.

Recently, we showed that exposure-based CBT delivered via the internet can be effective for severe health anxiety when tested against a basic control condition. ${ }^{12}$ However, this new therapy has not yet been compared with active treatment. The aim of the present study was therefore to investigate two equally credible internet-delivered treatments - exposure-based CBT v. BSM for severe health anxiety in a randomised controlled trial. We hypothesised that exposure-based CBT, in comparison with BSM, would lead to larger reductions of health anxiety.

\section{Method}

\section{Design}

This was a randomised controlled superiority trial in which 158 participants with severe health anxiety were randomised to exposure-based CBT $(n=79)$ (CBT group) or to BSM $(n=79)$ (BSM group) delivered via the internet in a 1:1 ratio without restriction. Duration of treatment was 12 weeks for both interventions and assessments with the primary outcome (the Health Anxiety Inventory, $\mathrm{HAI}^{13}$ ) were conducted at baseline, posttreatment and 6-month follow-up. The study was conducted in Stockholm, Sweden, and all participants provided informed consent. The regional ethics review board in Stockholm approved the study and the trial was registered at Clinicaltrials.gov (identifier: NCT01673035).

\section{Recruitment and sample}

Study participants were recruited nationally and information about the study was sent to psychiatrists and primary care physicians, and published on the study's website as well as in newspapers with national coverage. All potential participants had to apply through the study website portal. The inclusion criteria for the study were that participants had to:

(a) have a principal diagnosis of severe health anxiety (hypochondriasis) according to DSM-IV

(b) be at least 18 years old 
(c) have no ongoing or prior episode of bipolar disorder or psychosis

(d) have no ongoing substance misuse or addiction

(e) be on a stable dosage for at least 2 months if on antidepressant or anxiolytic medication and agree to keep the dosage constant throughout the study

(f) not have severe depressive symptoms or serious suicide ideation as indicated by a total score of $\geqslant 31$ or $\geqslant 4$ on item 9 of the Montgomery-Åsberg Depression Rating Scale - Selfrated (MADRS-S) ${ }^{14}$

(g) receive no concurrent psychological treatment for severe health anxiety and have no history of completed CBT for severe health anxiety during the past 3 years

(h) have no serious somatic disorder to which the health anxiety would be an adequate response.

Potential participants who applied to the study underwent a diagnostic interview with a licensed psychologist $(10 \%$ of assessments) or with a student in the final semester of the 5-year psychology programme at the Karolinska Institutet in Stockholm under the supervision of a licensed psychologist $(90 \%$ of assessments). Students had completed 3 years of study at Bachelor level and nearly 2 years at Masters level. The interview was conducted over the telephone, which has been shown to be a reliable administration format for structured psychiatric assessment. ${ }^{15}$ To establish whether diagnostic criteria for DSM-IV hypochondriasis were met, the Anxiety Disorders Interview Schedule $(\mathrm{ADIS})^{16}$ was used and the Mini International Neuropsychiatric Interview $(\mathrm{MINI})^{17}$ was used to assess other psychiatric Axis I disorders. Pre-treatment severity was assessed using the DSM-IV Global Assessment of Functioning (GAF) scale. All diagnostic assessors had received comprehensive training in conducting structured diagnostic interviews. There was no assessment of whether participants met diagnostic criteria for the new DSM-5 disorders somatic symptom disorder and illness anxiety disorder. ${ }^{18}$ Since the criteria for hypochondriasis are similar to those of somatic symptom disorder, it is likely that a large proportion of included participants would have met criteria for the latter disorder. Table 1 displays characteristics of the included participants.

\section{Outcome measures}

\section{Primary outcome measure}

The Health Anxiety Inventory (HAI $)^{13}$ was the primary outcome measure. The HAI has been shown to possess good psychometric qualities including high test-retest reliability $(r=0.90)$ and high internal consistency (Cronbach's $\alpha=0.95$ ). ${ }^{13}$

\section{Secondary outcome measures}

The Illness Attitude Scale (IAS) ${ }^{19}$ and the Whiteley Index (WI) ${ }^{20}$ were used as complimentary measures of health anxiety. We assessed depressive symptoms using the MADRS-S, ${ }^{14}$ and general anxiety and anxiety sensitivity using the Beck Anxiety Inventory $(\mathrm{BAI})^{21}$ and the Anxiety Sensitivity Index (ASI) ${ }^{22}$ respectively. Functional impairment was measured with the Sheehan Disability Scale (SDS), ${ }^{23}$ which assesses impairment in three domains: work, social and family life.

\section{Assessment of treatment credibility}

To assess treatment credibility, i.e. how believable the treatment is and how improved participants predict they will be, the C-scale ${ }^{24}$ was used. The C-scale has been shown to have high predictive value in the treatment of anxiety disorders. ${ }^{8}$

\section{Assessment of therapeutic working alliance and adverse events}

To assess whether participants' ratings of working alliance with their therapist differed between the treatment groups we used a revised version of the Working Alliance Inventory - Short-form (WAI-S), ${ }^{25}$ which measures three dimensions of working alliance: therapeutic bond, agreement on goals and agreement on tasks. From the 12 items of the original scale we used 6 items (items $1,5,6,7,8,11)$, two from each dimension. The chosen items were those judged most likely to be highly correlated with the full WAI-S scale.

At post-treatment, participants reported whether they had experienced any form of adverse event that they believed was due to the treatment they had received. This was done using a selfreport form (available from the authors on request). Participants were first asked to state whether any adverse event had occurred and, if so, to specify that event in a free-text comment.

\section{Randomisation and procedures}

Two measures were taken to prevent potential selection bias related to the randomisation procedure. First, a true random number service (www.random.org) was used for randomisation, and it was conducted by an external party not involved in the inclusion process. Second, allocation concealment was ensured through randomisation of all participants simultaneously after the decision of inclusion had been made. This meant that there was no possibility of having foreknowledge of forthcoming allocations. The assessments with the primary and secondary outcome measures

Table 1 Description of the participants

Exposure-based Behavioural stres CBT via internet management via $(n=79) \quad$ internet $(n=79)$

$\begin{array}{lll}\text { Gender } & & \\ \text { Women } & 64 & 61 \\ \text { Men } & 15 & 18\end{array}$

Age, years

Mean (s.d.) $41.7(13.6)$

Minimum-maximum 22-75 $21-70$

Severe health anxiety

Mean age at onset (s.d.)

GAF baseline score (s.d.)

Occupational status, $n$ (\%)

Working full time $37(47) \quad 39(49)$

Working part time

Pensioner or on sick leave

Student

Other

$18(23)$

9 (11)

9 (11)

6 (8)

Marital and parental status, $n$ (\%)

Married or de facto

$66(84)$

Parent, yes

58 (73)

$14.1(13.1)$

$13.0(13.1)$

$25.9(11.4)$

Pychiatric comorbidity, $n$ (\%)

Comorbid anxiety disorder

Comorbid major depression

26 (33)

$15(18)$

$9(11)$

$6(8)$

Stabilised psychotropic medication,

$n$ (\%)

SSRIS or SNRIS

11 (14)

$67(85)$

Benzodiazepines 
were conducted at baseline, post-treatment and at 6-month follow-up, and the C-scale was administered at weeks two and eight during treatment. Working alliance as measured with the WAI-S was assessed each week throughout treatment. Post-treatment, participants reported whether they had experienced any adverse events during the treatment phase. Additional assessments were conducted on a weekly basis to enable investigation of potential processes related to outcome but will be reported elsewhere. Participants completed assessments via the internet, which has been shown to be a reliable administration format. ${ }^{26}$

\section{Treatments}

\section{General description of internet-based psychological treatment}

Internet-based therapy, as used in both treatment arms in this study, can be described as therapist-guided online bibliotherapy. ${ }^{27}$ The general principle is that the patient should be exposed to the same interventions as if the treatment had been administered in a conventional format and the mechanisms for therapeutic change are thus thought to be the same. Internet-based treatment of this kind provides an excellent framework for comparing therapies with different content due to its firm structure. Treatment is structured as modules, which are the equivalent of sessions in conventional treatment, each comprising 5-15 pages of text and entail the necessary information for behaviour change as prescribed by the treatment. Each module has a set of homework assignments and throughout treatment patients have to complete these assignments and report to their therapists on how the work is progressing. The modules are fixed - that is, the content cannot be altered by the therapist or patient - which ensures high treatment integrity through control over which interventions the patient is exposed to. The therapist gives access to the next module, approximately one per week, when they think homework assignments have been adequately completed. Modules and homework assignments are designed to enable the patient and therapist to adapt the intervention to the patient's specific problems and behaviours. The modules and the therapist contact are provided through a secure internet-based platform and the patient has the same therapist throughout treatment. In general, therapist contact is strictly in the form of email-like communication, meaning that no real-time internet chat or telephone appointments are used.

\section{Internet-delivered exposure-based CBT}

The internet-delivered exposure-based CBT investigated in this trial was based on a model emphasising the role of negatively reinforced avoidance and safety behaviours as maintaining factors of health anxiety ${ }^{28,29}$ and has previously been shown to be effective when administered in both a conventional format ${ }^{30}$ and as internet-based treatment, ${ }^{12}$ with effects lasting at least 1 year after completed treatment. ${ }^{31}$ The main intervention was systematic exposure to health-anxiety-related situations or events in combination with response prevention. An example of this could be to trigger feared bodily sensations through physical exercise (exposure) while refraining from checking that the pulse is normal (response prevention). As a way to enhance exposure, especially to bodily sensations, mindfulness training was used. Mindfulness training was not used as a stand-alone intervention but as a way to increase the possibility that patients would conduct often highly anxiety-provoking exposure exercises and that they would not use distraction as a means to cope with worrying sensations. Thus, the treatment differed from, for example, the mindfulness-based cognitive therapy of McManus and colleagues, ${ }^{32}$ in which mindfulness training is the main component. As pointed out by Treanor, ${ }^{33}$ mindfulness training could facilitate extinction learning during exposure through increasing awareness of multiple conditioned triggers of anxiety. When conducting exposure and response prevention, patients were encouraged to have an accepting stance towards cognitions and emotions. The treatment thus used elements from the so-called third wave of CBT within an exposure-extinction paradigm.

Treatment was made up of 12 modules over 12 weeks and the content of each module is outlined in Table 2. The first six modules entailed all the main components of the treatment including rationale for and how to apply systematic exposure and response prevention. Modules 7-12 thus mainly served to motivate the patient to actively report on progress with the treatment in terms of conducted exposure. Seven therapists delivered the treatment and sent 17.0 messages each on average $($ s.d. $=6.9)$ to the participants. Three therapists were licensed psychologists with at least 3 years of experience in working with internet-based treatments and four therapists were students in their final semester of a 5-year psychology programme, who received supervision from a licensed psychologist. The students had extensive theoretical and clinical training at Bachelor as well as Masters level and were only a few months from graduating. The median therapist time spent weekly per patient was $11.0 \mathrm{~min}$.

\section{Internet-delivered BSM}

In contrast to the exposure-based CBT, the internet-delivered BSM was built on a model focusing on the reduction of health anxiety through direct control of distressing symptoms and through reduction of general stress. The main intervention was applied relaxation, which the patient learned to practice through a five-step programme. It also entailed several stress-management strategies including activity scheduling, structured problemsolving, and increasing recuperating activities. The treatment was similar to that developed by Salkovskis and colleagues, which has been found to be as effective and equally as credible as CBT in a randomised trial. ${ }^{5}$ Applied relaxation was inspired by but did not strictly follow the treatment developed by Öst, ${ }^{10}$ and has previously been tested as an internet-based intervention. ${ }^{34}$ The programme started with progressive relaxation, followed by release-only relaxation, conditioning a relaxed state to a verbal cue, and applying rapid relaxation in distressing situations. An important aspect of BSM was that patients were encouraged to use applied relaxation as a means of coping with health anxiety. However, it included no instructions to engage in exposure to health-anxiety-related situations and events. The general aim of BSM was that participants were trained to take control over their health anxiety symptoms by using different strategies, which contrasted to the exposure-based CBT where participants were encouraged to expose themselves to and accept health anxiety.

An overview of the treatment content is provided in Table 2. Treatment comprised of 12 modules and lasted for 12 weeks. Therapists sent 14.3 messages each on average (s.d. =6.2) to the participants. The treatment was carried out by the same seven therapists as for the exposure-based CBT. The median therapist time spent weekly per patient was $9.2 \mathrm{~min}$. This treatment was judged to be a highly suitable and a challenging comparator to exposure-based CBT, as it provided control over effects relating to: (a) presentation of an explicit psychological model for understanding health anxiety; (b) systematic and concrete behaviour change linked to the model; (c) therapeutic alliance; and as a consequence of (a)-(c), (d) treatment credibility.

\section{Statistical analysis}

Statistical analyses were conducted on an intention-to-treat basis using SPSS version 22.0 for Mac (SPSS IBM, Armonk). Continuous 


\begin{tabular}{|cll|}
\hline $\begin{array}{c}\text { Table } 2 \\
\text { Module }\end{array}$ & $\begin{array}{c}\text { Description of the main content of the two internet-delivered } \\
\text { Exposure-based CBT }\end{array}$ & $\begin{array}{l}\text { treatments } \\
\text { Behavioural stress management }\end{array}$ \\
\hline 1 & Introduction to CBT and mindfulness exercise & $\begin{array}{l}\text { Introduction to behavioural stress management, presentation of the } \\
\text { behavioural stress management model, applied relaxation, part I }\end{array}$ \\
\hline 2 & Presentation of the CBT model, continued mindfulness training & Applied relaxation, part II \\
\hline 3 & Cognitive processes, continued mindfulness training & Applied relaxation, part III \\
\hline 4 & Interoceptive exposure, continued mindfulness training & Applied relaxation, part IV \\
\hline 5 & Response prevention, continued mindfulness training & Applied relaxation, part V \\
\hline 7 & Exposure to health anxiety provoking stimuli & Stress management, continued applied relaxation \\
\hline 8 & Cxposure to illness thoughts & Stress management, continued applied relaxation \\
\hline 9 & Continued exposure and response prevention & Stress management, continued applied relaxation \\
\hline 10 & Continued exposure and response prevention & Stress management, continued applied relaxation \\
\hline 11 & A summary of the treatment & Stress management, continued applied relaxation \\
\hline 12 & Maintaining gains and relapse prevention & A summary of the treatment \\
\hline CBT, cognitive-behavioural therapy; SSRI, selective serotonin reuptake inhibitor. & Maintaining gains and relapse prevention \\
\hline
\end{tabular}

outcome data were analysed using linear mixed-effects model regression analysis for repeated measurements. This statistical method takes dependency in data into account by incorporating random effects (i.e. individual differences in intercept and slope) in the model in addition to the corresponding fixed effects, and provides unbiased parameters estimates when the data-set has missing values and reduces the risk of committing type 1 errors. ${ }^{35}$ Slope was modelled in three ways: (a) using all available data from baseline through post-treatment to 6-month follow-up; (b) from baseline to post-treatment; and (c) from post-treatment to 6-month follow-up. Treatment credibility was analysed using $t$-tests and nominal scale data with $\chi^{2}$-tests. Analysis of missing data patterns were conducted using Little's missing completely at random test. ${ }^{36}$ Effect sizes (Cohen's $d$ ) were calculated using pooled standard deviations.

\section{Results}

\section{Attrition and adherence}

Figure 1 displays the participant flow through the trial. Of the 158 participants, 158 (100\%) completed outcome measures at baseline, $151(96 \%)$ at post-treatment and $142(90 \%)$ at 6-month follow-up. Little's test showed that data were missing completely at random and thus were unrelated to the observed variables, including type of treatment $\left(\chi^{2}=321.6\right.$; d.f. $\left.=289 ; P=0.09\right)$. The CBT group completed 8.5 modules on average $(\mathrm{s} . \mathrm{d}=3.3)$ out of a possible total of 12 , and 62 participants $(78 \%)$ completed at least 6 modules and were therefore considered treatment completers. The average number of completed modules in the BSM group was 8.3 (s.d. $=3.3$ ) of 12 , and 62 participants $(78 \%)$ completed at least 6 modules. In the CBT group, 72 (91\%) participants completed at least four modules and thus initiated systematic exposure. There were no significant differences between the groups regarding completed modules ( $t=0.3$; d.f. $=156 ; P=0.66)$. In both groups there was a significant association between number of completed modules and baseline to post-treatment change score on the primary outcome HAI $(r=0.27-0.30 ; P<0.04)$.

\section{Treatment credibility and working alliance}

There was no significant difference between the groups on the C-scale at weeks 2 or $8(t=0.5-1.6$; d.f. $=1,151-155 ; P=0.76-$ $0.23)$, i.e. there was no significant difference in terms of how credible the participants regarded the respective treatments and how much they expected to be improved by them. At week two the average C-scale score was 36.1 (s.d.=8.8) in the CBT group and 36.0 (s.d.=9.7) in the BSM group. The corresponding estimates at week 8 were 35.6 (s.d. $=9.5)$ and 33.1 (s.d.=10.5). Participants' ratings of working alliance with the WAI-S did not
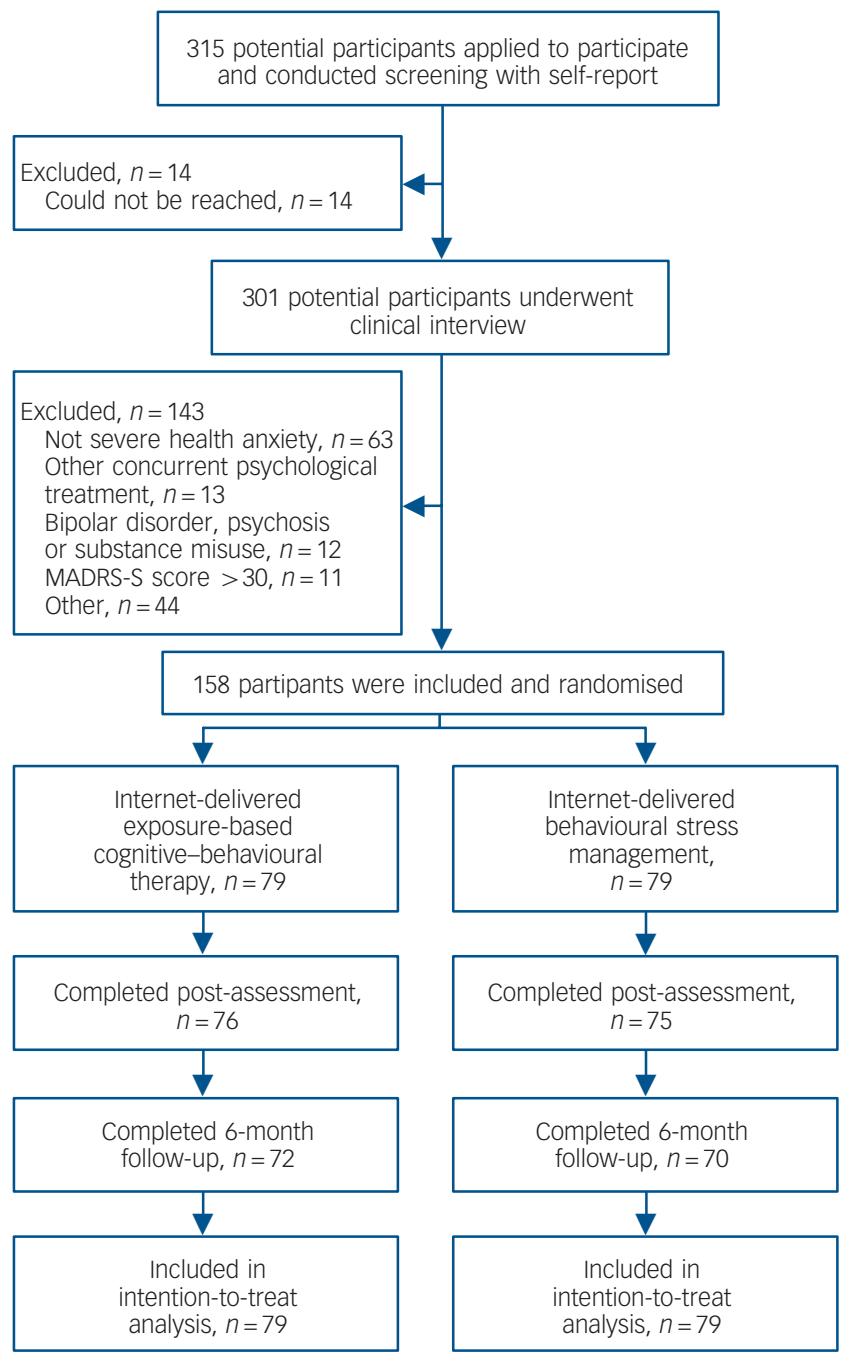

Fig. 1 Participant flow through the trial. Montgomery-Åsberg Depression Rating Scale - Self-rated. 
significantly differ between the treatment groups at any time on the weekly assessments throughout treatment $(t=0.2-1.5$; d.f. $=1,109-144 ; P=0.83-0.15)$.

\section{Primary outcome measure}

Mixed-effects models analyses showed a significant interaction effect of group and time across the entire assessment period (i.e. from baseline to 6-month follow-up), indicating superior improvements in exposure-based CBT compared with BSM $(F=3.9$; d.f. $=2,121 ; P=0.022)$. There was also a significant interaction effect from baseline to post-treatment $(F=6.8$; d.f. $=1,149$; $P=0.01$ ), indicating larger improvements in exposure-based CBT. There was no significant interaction effect from post-treatment to 6-month follow-up ( $F=0.9$; d.f. $=1,140 ; P=0.35)$. There was no effect of time from post-treatment to 6-month follow-up, suggesting stability of gains made at post-treatment $(F=1.8$; d.f. $=1,140 ; P=0.18)$. Health anxiety was reduced in both treatment groups as indicated by significant main effects of time from baseline to post-treatment and to 6-month follow-up within each group $(F=263.8-88.7$; d.f. $=1-2,65-87 ; P<0.001)$. Table 3 displays means and effect sizes on all outcome measures. As shown in Table 3, both treatment groups made large improvements on the primary outcome measure, whereas between-group effect sizes were small at post-treatment and 6-month follow-up.

\section{Secondary outcome measures}

The same outcome pattern was shown for the complementary health anxiety measure IAS and for depressive symptoms as assessed with the MADRS-S, i.e. significant interaction effects of group and time from baseline to post-treatment and 6-month follow-up, indicating larger improvements in the CBT group than in the BSM group $\left(F=7.2-3.9\right.$; d.f. $=1-2,121-188 ; P^{\prime} s=0.008-$ $0.033)$. There was a significant main effect of time from baseline to post-treatment and to 6-month follow-up ( $F=155.6-23.0$; d.f. $\left.=1-2,60-100 ; P^{\prime} s<0.001\right)$ on the IAS and MADRS-S within each group, but no significant interaction or main effects from post-treatment to 6-month follow-up $(F=3.5-0.0$; d.f. $=1,137-140$; $P=0.06-0.84)$. Within-group effect sizes were large on the IAS in both groups (Table 3), whereas the CBT group made large improvements on the MADRS-S compared with moderate improvements in the BSM group, and between-group effect sizes were small on both measures. On the BAI (general anxiety), ASI (anxiety sensitivity) and SDS (functional impairment) there were significant within-group improvements from baseline to post-treatment and to 6-month follow-up in both groups $(F=99.9-4.4 ; \quad$ d.f. $=1-2, \quad 75-93 ; \quad P=0.001-0.014), \quad$ but no significant interaction effects of group and time $(F=2.3-0.8$; d.f. $=1-2,149-188 ; P=0.13-0.46)$. On these measures there was no effect of time or time $\times$ group interaction from posttreatment to 6-month follow-up $(F=1.9-0.0$; d.f. $=1,138-141$; $P=0.16-0.90)$, except on the ASI where there was a significant main effect of time $(F=07.8$; d.f. $=1,141 ; P=0.006)$, indicating improvement in both groups after end of therapy. As shown in Table 3, baseline to post-treatment and to 6-month follow-up effect sizes on the BAI were moderate to large in the CBT group and moderate in the BSM group. On the ASI and SDS, withingroup effect sizes in the CBT group were moderate, whereas the BSM group made small to moderate improvements on the ASI and small improvements on the SDS.

\section{Adverse events}

At post-treatment, participants reported the presence of adverse events during the treatment. However, analyses showed that there was no significant difference between the groups in terms of adverse events $\left(\chi^{2}=0.6\right.$; d.f. $\left.=1 ; P=0.48\right)$. In the CBT group, 63 participants $(84 \%)$ reported no adverse event and 12 participants (12\%) did experience an adverse event (4 participants did not

Table 3 Means and effect sizes $(d)$ on primary and secondary outcome measures

\begin{tabular}{|c|c|c|c|c|c|c|c|}
\hline \multirow[b]{3}{*}{ Measure (scale range) } & \multirow{3}{*}{$\begin{array}{c}\text { Pre } \\
\text { Mean (s.d.) }\end{array}$} & \multirow{3}{*}{$\begin{array}{c}\text { Post } \\
\text { Mean (s.d.) }\end{array}$} & \multirow{3}{*}{$\begin{array}{l}\text { 6-month follow-up } \\
\text { Mean (s.d.) }\end{array}$} & \multicolumn{4}{|c|}{ Effect size } \\
\hline & & & & \multicolumn{2}{|c|}{ Within group } & \multicolumn{2}{|c|}{ Between group } \\
\hline & & & & Pre-Post & Pre-follow-up & Post & 6-month follow-up \\
\hline \multicolumn{8}{|l|}{ Primary outcome } \\
\hline HAl (0-192) & & & & & & 0.26 & 0.13 \\
\hline I-CBT & $105.3(17.7)$ & $65.7(26.1)$ & $64.5(26.4)$ & 1.78 & 1.83 & & \\
\hline I-BSM & $102.9(22.4)$ & $72.6(26.1)$ & $68.1(27.0)$ & 1.22 & 1.41 & & \\
\hline \multicolumn{8}{|l|}{ Secondary outcomes } \\
\hline IAS (0-108) & & & & & & 0.47 & 0.32 \\
\hline I-CBT & $67.5(11.1)$ & $47.1(15.6)$ & $46.4(15.5)$ & 1.51 & 1.58 & & \\
\hline I-BSM & $68.6(12.8)$ & $54.1(14.2)$ & $51.2(14.6)$ & 1.07 & 1.27 & & \\
\hline WI (0-14) & & & & & & 0.20 & 0.17 \\
\hline I-CBT & $10.6(2.1)$ & $7.2(3.5)$ & $6.8(3.7)$ & 1.18 & 1.28 & & \\
\hline I-BSM & $10.5(2.3)$ & $7.9(3.4)$ & $7.4(3.4)$ & 0.90 & 1.08 & & \\
\hline MADRS-S (0-54) & & & & & & 0.05 & 0.05 \\
\hline I-CBT & $12.8(7.0)$ & $7.7(6.1)$ & $7.1(5.3)$ & 0.78 & 0.91 & & \\
\hline I-BSM & $10.8(6.2)$ & $8.0(6.5)$ & $7.4(6.0)$ & 0.44 & 0.56 & & \\
\hline BAI (0-63) & & & & & & 0.10 & 0.13 \\
\hline I-CBT & $18.4(8.2)$ & $12.3(7.4)$ & $11.1(7.0)$ & 0.78 & 0.95 & & \\
\hline I-BSM & $18.3(9.0)$ & 13.1 (9.3) & $12.2(7.8)$ & 0.57 & 0.72 & & \\
\hline ASI (0-64) & & & & & & 0.43 & 0.33 \\
\hline I-CBT & $22.0(11.2)$ & $15.6(9.0)$ & $14.4(8.6)$ & 0.63 & 0.76 & & \\
\hline I-BSM & $24.3(10.8)$ & $19.9(10.8)$ & $17.4(9.8)$ & 0.41 & 0.67 & & \\
\hline SDS (0-30) & & & & & & 0.00 & -0.05 \\
\hline I-CBT & 9.5 (7.1) & $6.0(6.5)$ & $5.9(5.8)$ & 0.51 & 0.55 & & \\
\hline I-BSM & $8.0(6.4)$ & $6.0(5.3)$ & $5.6(5.9)$ & 0.34 & 0.39 & & \\
\hline
\end{tabular}


complete the form). In the BSM group, 62 participants (89\%) had no adverse events and $8(11 \%)$ participants did (9 participants did not complete the form). In both treatment groups the most commonly reported adverse event was increased levels of anxiety in the early phase of therapy. There was no report of serious adverse events, i.e. events leading to acute health risks demanding admission to hospital.

\section{Discussion}

\section{Main findings}

The aim of this study was to investigate the effects of exposurebased CBT delivered via the internet in comparison with an active treatment based on applied relaxation and stress management (BSM) for severe health anxiety. As predicted, the results showed that both groups made large within-group improvements on the primary outcome measure, but participants receiving exposurebased CBT had significantly larger reductions of health anxiety. There was no significant difference between the treatments in terms of treatment credibility, suggesting that exposure-based CBT gives an effect beyond what could be expected from taking part in a credible, coherent and comprehensive psychological treatment.

\section{General and specific effects and comparison to prior studies}

To our knowledge this is the second study to investigate exposurebased CBT delivered via the internet for severe health anxiety. In the first study of this type, the treatment was found to be highly effective in comparison with a basic control condition that did not receive active treatment. ${ }^{12}$ We therefore considered it important to investigate the effects of exposure-based CBT, controlling for important factors such as being presented with a model for understanding health anxiety, making behavioural changes aimed at reducing health anxiety, therapeutic alliance and the expectation of improvement. It is important to highlight that this study did not have experimental control over exposure and response prevention as isolated intervention, as that would have required that participants in the control treatment to receive the exact same treatment minus exposure and response prevention. Considering that exposure is the basis of treatment, we judged it as close to impossible to create a comparator of this kind (i.e. exposure-based CBT minus exposure and nothing more) that would still be equally credible to the participants, which was why BSM was used. We also considered it ethically questionable to administer a treatment that would control for only non-specific effects as that would have required exposing participants to an invalid treatment but describing it as effective CBT for severe health anxiety. By using BSM, exposure-based CBT was put to one of the toughest tests possible, considering that BSM and applied relaxation have previously been shown to be effective in a conventional format for severe health anxiety and general anxiety respectively. ${ }^{5,8}$ In addition, both treatments had the same structure in terms of explicit presentation of the rationale for treatment, concrete behaviour change and homework assignments. Thus, the main difference was that of exposure to $v$. control over symptoms, where the treatment focusing on the former perspective produced larger reductions in health anxiety.

In light of the above, it would not be adequate to describe BSM as merely psychological placebo and the observed results in terms of small to moderate group differences in improvement rates between treatments as therefore expected. Nevertheless, it should be pointed out that BSM was effective with large effect sizes that were nearly identical to that with BSM when tested for severe health anxiety in a face-to-face format by Clark and colleagues. ${ }^{5}$ As for exposure-based CBT, the large within-group effect size on the primary outcome found in the previous randomised trial ${ }^{12}$ was replicated in this study. When comparing within-group effects with CBT in a conventional format, the internet-delivered treatment showed results on par with those of conventional CBT. For example, in terms of health anxiety (measured as 'time seriously worried about health'), the pre-topost-treatment effect size (CBT group) in the study by Clark et al was Cohen's $d 1.80,{ }^{5}$ which again is nearly identical to the improvement on the primary outcome in our CBT group $(d=1.78)$. In a study by Visser and colleagues ${ }^{9}$ investigating exposure and response prevention for severe health anxiety in a conventional format, the pre-to-post-treatment effect size on the IAS was $d=1.14$ compared with $d=1.51$ on the same measure in the present study. Although comparisons across studies should be done with caution, this suggests that internet-delivered exposure-based CBT tested in this trial has the potential to be as effective as the empirically supported treatments administered in a conventional format.

\section{Mechanisms}

What made exposure-based CBT effective? In terms of treatment procedures, the most important factor affecting health anxiety mechanisms is likely to be the behaviour change itself; that is, confronting situations that elicit health anxiety and maintaining contact with adverse thoughts and emotions. However, one important question is why this would have an impact on health anxiety. As pointed out by Moscovitch et al, the anxiety-reducing effect of exposure is probably best explained by extinction through new learning ${ }^{37}$ - conditioned stimuli (e.g. bodily sensations) become associated with safety rather than danger, a process that can rely on several cognitive systems. In a recently conducted mediational study, we found that exposure-based CBT for severe health anxiety led to less attention to bodily symptoms, reduced perceived risk of disease and reduced intolerance of uncertainty, three factors that in turn were associated with outcome and mediated the effect of the treatment. ${ }^{38}$ Thus it seems that engaging in exposure and response prevention and using mindfulness to enhance exposure leads to important effects on attentional and cognitive processes that play a role in reducing health anxiety. Future studies should compare the relative effects of exposure with other treatment interventions on these health anxiety mechanisms.

\section{Clinical implications}

There are several clinical implications. First, the results suggest that exposure-based CBT delivered via the internet for people with severe health anxiety can be effective and yield large reductions in health anxiety. As therapists in internet-based CBT can have up to 80 patients in treatment simultaneously, ${ }^{39}$ this treatment has the potential to increase accessibility to psychological treatment for severe health anxiety and to be cost-effective compared with face-to-face treatment. A previous study showed that internetdelivered exposure-based CBT for severe health anxiety can be highly cost-effective in comparison to no treatment and that there is a net societal cost gain for each incremental quality adjusted life year produced by the treatment. ${ }^{31}$ This study supports the clinical use of exposure and response prevention for this patient group, as this method is superior compared with an active and credible internet-delivered treatment based on applied relaxation and stress management. However, as participants receiving BSM made large improvements, it should be highlighted that improvements can be made also through a treatment focusing on direct symptom 
control. Although the larger improvements found in exposurebased CBT promotes its use as the treatment of choice, we regard $\mathrm{BSM}$ as a treatment option that can be considered for patients who would be judged unsuitable for exposure-based CBT. We do not regard exposure-based CBT to be unsuitable for patients with severe symptoms, which is supported by results from a previous study of predictors of exposure-based CBT showing that higher levels of baseline health anxiety were associated with larger improvements after treatment. ${ }^{40}$

Regarding module completion, participants on average completed about two-thirds of the modules, which deserves comment. The modules are designed so that the most important information is provided in the first six modules and thereafter modules to a large extent serve the purpose of being the natural means for maintaining the contact between therapist and patient. Against this background, it is expected that effect sizes can be large despite not all modules being completed. It could be that the treatment would benefit from being reduced to six to eight modules. However, our previous experience is that it can be difficult to motivate the patient to keep reporting on treatment work for 12 weeks if they have no reinforcement in terms of access to new modules after just half that time.

An important venue for future research is to investigate moderators, i.e. predictors that have an interaction effect with treatment type on the outcome. Also, an important question to address is how well the internet-delivered exposure-based CBT tested in our study compares with the same treatment when provided in a conventional format. Finally, it would be valuable to see whether the effect of the treatment is maintained when given to participants with the DSM-5 diagnoses somatic symptom disorder and illness anxiety disorder, ${ }^{18}$ which replace DSM-IV hypochondriasis. As the hypochondriasis criteria used in our study are more similar to the somatic symptom disorder criteria than to illness anxiety disorder, it is possible that the findings of the present study to a large extent are generalisable to individuals with somatic symptom disorder.

\section{Strengths and limitations}

Central strengths of the study include the randomised design, the thoroughly assessed sample, the use of well-validated outcome measures, low attrition rates, and high statistical power. Also, internet-based treatment is a highly suitable model for conducting this kind of comparative study as there is a high control over the treatment material that the participant is exposed to: the firm structure with modules as a core feature makes contamination between treatments close to impossible. One aspect of the study that to some extent was both a strength and a limitation was that the same therapists provided the therapeutic support in both treatment groups. The advantage of this was that factors related to the therapists were kept constant across treatments, but the potential disadvantage is that it cannot be ruled out that therapists believed more in one treatment than the other. The ideal design for handling this aspect would have been to recruit an equal amount of therapists specialised in internet-based CBT and internet-based BSM and to train them in their non-specialty therapy and let each therapist deliver as many internet-based CBT as internet-based BSM treatments. However, as both treatments provided in this study are new, this was not possible. Importantly, the fixed module structure of internet-based treatments leaves less room for impact of the therapist on outcome.

Regarding limitations, it should be noted that we did not use a waiting list control group. This means that it is possible that participants would have improved just as much even if had they had not received any treatment. Considering the chronicity of severe health anxiety ${ }^{2}$ and that the within-group effect size of the untreated control condition was $d=0.19$ (pre-to post-treatment) in the previous randomised controlled trial testing exposure-based CBT delivered via the internet, ${ }^{12}$ we regard spontaneous remission as an unlikely explanation of the improvements found in the present trial. In addition, improvements related to factors outside the trial would not explain the significant interaction effects of treatment and time. Another limitation is that we did not assess diagnostic status at post-treatment and 6-month follow-up. However, as a comparison, our CBT group made similar improvements on the primary outcome HAI as the corresponding group in the previous randomised controlled trial, in which two-thirds did not meet diagnostic criteria for severe health anxiety at post-treatment. ${ }^{12}$

Despite these limitations, our results indicate that exposurebased CBT delivered via the internet can be an effective treatment for severe health anxiety that yields treatment effects above and beyond those of an active, credible and effective internet-delivered psychological treatment based on applied relaxation and stress management. This study gives further support for using internet-delivered exposure-based CBT as a means for increasing accessibility to effective psychological treatment for severe health anxiety.

Erik Hedman, PhD, Department of Clinical Neuroscience, Osher Center for Integrative Medicine, and Department of Clinical Neuroscience, Division of Psychology, Karolinska Institutet, Stockholm; Erland Axelsson, MSc, Anders Görling, MSC, Carina Ritzman, MSC, Markus Ronnheden, MSC, Department of Clinical Neuroscience, Division of Psychology, Karolinska Institutet, Stockholm; Samir El Alaoui, MSc, Erik Andersson, MSc, Department of Clinical Neuroscience, Division of Psychiatry, Karolinska Institutet, Stockholm; Mats Lekander, PhD, Department of Clinical Neuroscience, Osher Center for Integrative Medicine, Karolinska Institutet, Stockholm, and Stress Research Institute, Stockholm University, Stockholm; Brjánn Ljótsson, PhD, Department of Clinical Neuroscience, Division of Psychology, Karolinska Institutet, Stockholm, Sweden

Correspondence: Dr Erik Hedman, Department of Clinical Neuroscience, Karolinska Institutet, Retzius väg 8, SE-171 77 Stockholm, Sweden. Email: kire.hedman@ki.se

First received 24 Oct 2013, final revision 4 Mar 2014, accepted 2 May 2014

\section{References}

1 American Psychiatric Association. Diagnostic and Statistical Manual of Mental Disorders (Fourth Edition) (DSM-IV). APA, 1994.

2 Barsky AJ, Fama JM, Bailey ED, Ahern DK. A prospective 4- to 5-year study of dsm-iii-r hypochondriasis. Arch Gen Psychiatry 1998; 55: 737-44.

3 Tyrer P, Cooper S, Crawford M, Dupont S, Green J, Murphy D, et al. Prevalence of health anxiety problems in medical clinics. J Psychosom Res 2011; 71: 392-4.

4 Sunderland M, Newby JM, Andrews G. Health anxiety in australia: prevalence, comorbidity, disability and service use. Br J Psychiatry 2013; 202: $56-61$.

5 Clark DM, Salkovskis PM, Hackmann A, Wells A, Fennell M, Ludgate J, et al. Two psychological treatments for hypochondriasis. A randomised controlled trial. Br J Psychiatry 1998; 173: 218-25.

6 Barsky AJ, Ahern DK. Cognitive behavior therapy for hypochondriasis: a randomized controlled trial. JAMA 2004; 291: 1464-70.

7 Tyrer $\mathrm{P}$, Cooper S, Salkovskis $\mathrm{P}$, Tyrer H, Crawford M, Byford S, et al. Clinical and cost-effectiveness of cognitive behaviour therapy for health anxiety in medical patients: a multicentre randomised controlled trial. Lancet 2014; 383: 219-25.

8 Borkovec TD, Costello E. Efficacy of applied relaxation and cognitivebehavioral therapy in the treatment of generalized anxiety disorder. J Consult Clin Psychol 1993; 61: 611-9.

9 Visser S, Bouman TK. The treatment of hypochondriasis: exposure plus response prevention vs cognitive therapy. Behav Res Ther 2001; 39 : 423-42.

10 Öst LG. Applied relaxation: Description of a coping technique and review of controlled studies. Behav Res Ther 1987; 25: 397-409. 
11 Sorensen $\mathrm{P}$, Birket-Smith $\mathrm{M}$, Wattar U, Buemann I, Salkovskis $\mathrm{P}$ A randomized clinical trial of cognitive behavioural therapy versus short-term psychodynamic psychotherapy versus no intervention for patients with hypochondriasis. Psychol Med 2011; 41: 431-41.

12 Hedman E, Andersson G, Andersson E, Ljotsson B, Ruck C, Asmundson GJ, et al. internet-based cognitive-behavioural therapy for severe health anxiety: Randomised controlled trial. Br J Psychiatry 2011; 198: 230-6.

13 Salkovskis PM, Rimes KA, Warwick HM, Clark DM. The health anxiety inventory: Development and validation of scales for the measurement of health anxiety and hypochondriasis. Psychol Med 2002; 32: 843-53.

14 Svanborg P, Åsberg M. A new self-rating scale for depression and anxiety states based on the comprehensive psychopathological rating scale. Acta Psychiatr Scand 1994; 89: 21-8.

15 Rohde P, Lewinsohn PM, Seeley JR. Comparability of telephone and face-toface interviews in assessing Axis I and II disorders. Am J Psychiatry 1997; 154: 1593-8.

16 Di Nardo PA, O'Brien GT, Barlow DH, Waddell MT, Blanchard EB. Reliability of DSM-III anxiety disorder categories using a new structured interview. Arch Gen Psychiatry 1983; 40: 1070-4.

17 Sheehan DV, Lecrubier $\mathrm{Y}$, Sheehan $\mathrm{KH}$, Amorim $\mathrm{P}$, Janavs J, Weiller $\mathrm{E}$, et al. The Mini-International Neuropsychiatric Interview (M.I.N.I.): the development and validation of a structured diagnostic psychiatric interview for DSM-IV and ICD-10. J Clin Psychiatry 1998; 59 (suppl 20): 22-33.

18 American Psychiatric Association. Diagnostic and Statistical Manual of Mental Disorders (5th edn) (DSM-5). APA, 2013.

19 Speckens AEM, Spinhoven P, Sloekers PP, Bolk JH, van Hemert AM. A validation study of the Whitely Index, the Illness Attitude Scales, and the Somatosensory Amplification Scale in general medical and general practice patients. J Psychosom Res 1996; 40: 95-104.

20 Pilowsky I. Dimensions of hypochondriasis. Br J Psychiatry 1967; 113: 89-93.

21 Beck AT, Epstein N, Brown G, Steer RA. An inventory for measuring clinica anxiety: psychometric properties. J Consult Clin Psychol 1988; 56: 893-7.

22 Reiss S, Peterson RA, Gursky DM, McNally RJ. Anxiety sensitivity, anxiety frequency and the prediction of fearfulness. Behav Res Ther 1986; 24: 1-8.

23 Sheehan DV. The Anxiety Disease. Scribner, 1983.

24 Borkovec TD, Nau SD. Credibility of analogue therapy rationales. J Behav The Exp Psychiatry 1972; 3: 257-60.

25 Tracey TJ, Koktovic AM. Factor structure of the working alliance inventory. Psychol Assess 1989; 1: 207-10.

26 Hedman E, Ljótsson B, Rück C, Furmark T, Carlbring P, Lindefors N, et al. internet administration of self-report measures commonly used in research on social anxiety disorder: a psychometric evaluation. Comput Human Behav 2010; 26: 736-40.
27 Hedman E, Ljótsson B, Lindefors N. Cognitive behavior therapy via the internet: a systematic review of applications, clinical efficacy and costeffectiveness. Expert Rev Pharmacoecon Outcomes Res 2012; 12: 745-64.

28 Furer $\mathrm{P}$, Walker JR. Treatment of hypochondriasis with exposure. J Contemp Psychother 2005; 35: 251-67.

29 Furer $\mathrm{P}$, Walker JR, Stein MB. Treating Health Anxiety and Fear of Death A Practitioner's Guide. Springer, 2007.

30 Hedman E, Ljótsson B, Andersson E, Rück C, Andersson G, Lindefors N. Effectiveness and cost offset analysis of group cbt for hypochondriasis delivered in a psychiatric setting: an open trial. Cogn Behav Ther 2010; 39 239-50.

31 Hedman E, Andersson E, Lindefors N, Andersson G, Ruck C, Ljotsson B. Cost-effectiveness and long-term effectiveness of internet-based cognitive behaviour therapy for severe health anxiety. Psychol Med 2013; 43: 363-74.

32 McManus F, Surawy C, Muse K, Vazquez-Montes M, Williams JM. A randomized clinical trial of mindfulness-based cognitive therapy versus unrestricted services for health anxiety (hypochondriasis). J Consult Clin Psychol 2012; 80: 817-28.

33 Treanor $\mathrm{M}$. The potential impact of mindfulness on exposure and extinction learning in anxiety disorders. Clin Psychol Rev 2011; 31: 617-25.

34 Ljotsson B, Hedman E, Andersson E, Hesser H, Lindfors $P$, Hursti $T$, et al. internet-delivered exposure-based treatment vs. Stress management for irritable bowel syndrome: a randomized trial. Am J Gastroenterol 2011; 106: 1481-91.

35 Muthén B, Curran PJ. General longitudinal modeling of individual differences in experimental designs: a latent variable framework for analysis and power estimation. Psychol Methods 1997; 2: 371-402.

36 Little RJA, Rubin DB. Statistical Analysis with Missing Data. Wiley, 1987.

37 Moscovitch DA, Antony MM, Swinson RP. Exposure-based treatments for anxiety disorders: theroy and process. In Oxford Handbook of Anxiety and Related Disorders (eds MM Antony, MB Stein): 461-75. Oxford University Press, 2008.

38 Hedman E, Andersson E, Andersson G, Lindefors N, Lekander M, Ruck C, et al. Mediators in internet-based cognitive behavior therapy for severe health anxiety. PLOS One 2013; 8: e77752.

39 Hedman E, Ljotsson B, Ruck C, Bergstrom J, Andersson G, Kaldo V, et al. Effectiveness of internet-based cognitive behaviour therapy for panic disorder in routine psychiatric care. Acta Psychiatr Scand 2013; 128: 457-67.

40 Hedman E, Lindefors N, Andersson G, Andersson E, Lekander M, Ruck C, et al. Predictors of outcome in internet-based cognitive behavior therapy for severe health anxiety. Behav Res Ther 2013; 51: 711-7. 